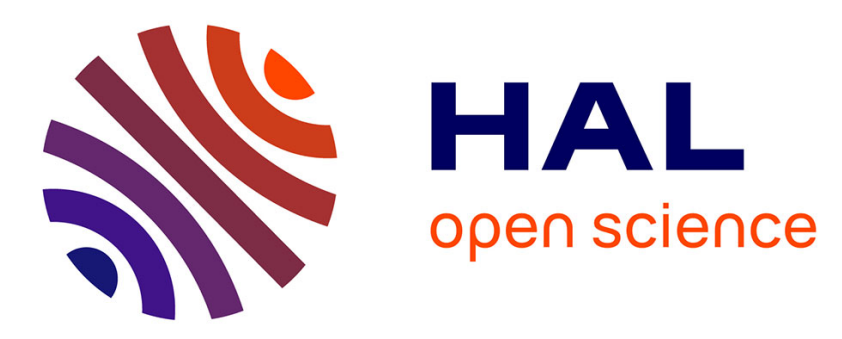

\title{
Contrôle actif des structures appliqué à une structure souple
}

Michel Vergé, Nazih Mechbal, Gérard Coffignal

\section{To cite this version:}

Michel Vergé, Nazih Mechbal, Gérard Coffignal. Contrôle actif des structures appliqué à une structure souple. Journal Européen des Systèmes Automatisés (JESA), 2003, 37, pp.501-526. 10.3166/jesa.37.501-526 . hal-00021081

\section{HAL Id: hal-00021081 https://hal.science/hal-00021081}

Submitted on 26 Mar 2018

HAL is a multi-disciplinary open access archive for the deposit and dissemination of scientific research documents, whether they are published or not. The documents may come from teaching and research institutions in France or abroad, or from public or private research centers.
L'archive ouverte pluridisciplinaire HAL, est destinée au dépôt et à la diffusion de documents scientifiques de niveau recherche, publiés ou non, émanant des établissements d'enseignement et de recherche français ou étrangers, des laboratoires publics ou privés. 


\title{
Contrôle actif des structures appliqué à une structure souple
}

\author{
Michel Vergé - Nazih Mechbal — Gérard Coffignal
}

\author{
École Nationale Supérieure d'Arts et Métiers \\ Laboratoire de Mécanique des Structures et des Procédés (LMSP) \\ 151, bd de l'hôpital \\ F-75013 Paris \\ Michel.Verge@paris.ensam.fr
}

RESUME Le contrôle actif des structures mécaniques souples est un champ de recherches très intense. Dans cet article, nous traitons le rejet de perturbations par des simulations puis nous validons les méthodes par des expérimentations sur une structure de laboratoire. Cette structure a été modélisée par éléments finis puis identifiée par des techniques fréquentielles. Elle est réalisée à partir d'une feuille d'aluminium pliée d'épaisseur $0,5 \mathrm{~mm}$. Les capteurs et actionneurs sont constitués par des paires de matériau piézo-électrique collées sur les faces opposées de la feuille. Pour des perturbations périodiques, le rejet de perturbation est obtenu avec un correcteur mixte qui est composé de deux parties : un correcteur robuste et un correcteur adaptatif.

ABSTRACT. Active control is a challenging research field on flexible mechanical structures. This paper is concerned with simulations of reject perturbations and with experiments on $a$ laboratory test bed. This structure has been first modeled by finite elements and then identified by frequential methods. It is made of aluminum sheet with $0.5 \mathrm{~mm}$ of thickness. Sensors and actuators are twin patches of piezoelectric material bounded onto the opposite faces of the sheet. For periodic disturbances, rejection is obtained by a mixed controller which is build with two parts: a robust controller and an adaptative controller.

MOTS-CLÉs : contrôle actif, rejet de perturbations, matériau piézo-électrique, éléments finis. KEYWORDS: Active control, disturbance rejection, piezoelectric material, finite elements. 


\section{Introduction}

Les avancées technologiques de ces dernières années ont vu apparaître l'émergence de nouveaux matériaux appelés «matériaux intelligents». Ces matériaux doivent leur nom à leur capacité de modifier spontanément leurs propriêtés physiques (forme, conductivité, couleur, etc.) en réponse à des sollicitations naturelles ou provoquées. On peut citer parmi eux les matériaux piézoélectriques, électrostrictifs, photochromes ou encore les matériaux à mémoire de forme.

La recherche constante de mécanismes de plus en plus performants, répondant à des exigences de coût et donc à une optimisation de la masse, a donné naissance aux premières applications de ces matériaux. Utilisés comme actionneurs ou comme capteurs et associés à une loi de commande, ils permettent de construire des structures dites «intelligentes» ou «actives». Le mécanisme est alors doté d'une chaîne d'acquisition permettant de mesurer son état vibratoire, d'actionneurs donnant la possibilité de réagir en conséquence et une structure de commande faisant la jonction entre ces deux entités. Ce dispositif est communément appelé « contrôle actif ».

Un des objectifs du contrôle actif des structures est celui de l'amortissement de leurs vibrations. En effet, l'allégement des structures s'accompagne souvent d'une plus grande flexibilité et inévitablement d'une modification de leurs caractéristiques vibratoires (modes propres: fréquences et formes). Par exemple, dans le contexte des véhicules automobiles, ces vibrations peuvent provoquer des nuisances sonores inacceptables. De même, pour les structures supportant des systèmes de mesures des déplacements relatifs, ces vibrations peuvent conduire à des mesures erronées.

De nombreuses publications couvrent plusieurs aspects de ces recherches que l'on classe en deux catégories de commande :

- les méthodes adaptatives utilisées dans un contexte de commande par anticipation, en supposant que la perturbation est mesurable [MAC 95, KUO 96, WID 85],

- les méthodes robustes utilisées pour déterminer une boucle de retour sur les mesures, en supposant que la perturbation n'est pas mesurable [GAW 98, LEE 02].

Notons que la plupart de ces méthodes ne concernent que les systèmes monovariables. En pratique, l'amortissement actif des structures flexibles soulève plusieurs difficultés qui se résument en :

- difficulté de modélisation : en effet les matériaux utilisés ne sont pas forcément homogènes, les formes issues de pliages ne sont pas exactement connues, les collages des capteurs et actionneurs ne sont pas parfaits, etc.

- difficulté de définir les emplacements idéaux des actionneurs et des capteurs.

- difficulté de réduire le modèle initialement délivré par une « modélisation par éléments finis» en un modèle utilisable pour la synthèse d'une loi de commande ; 
- difficulté de réaliser un correcteur efficace et robuste vis-à-vis des erreurs introduites par la modélisation et la réduction de modèle.

De nombreux auteurs ont étudié les points précédents (se référer [HEN 00], pour une bibliographie détaillée). L'originalité de cet article réside dans la présentation d'une méthodologie complète de mise en aeuvre du contrôle actif des vibrations sur une structure flexible munie de capteurs et actionneurs piézo-électriques. Nous donnons ainsi la modélisation par éléments finis que nous comparons ou modèle obtenu par identification expérimentale. De plus, pour les systèmes multivariables nous développons une « méthode mixte " qui associe la méthode adaptative et une commande robuste. Et enfin, nous présentons les résultats expérimentaux effectivement réalisés sur un dispositif de laboratoire.

\section{Présentation et modélisation du procédé expérimental}

\subsection{Dispositif expérimental}

Afin d'illustrer l'implantation du contrôle actif par éléments piézo-électriques, une structure nommée CASYM a été réalisée.
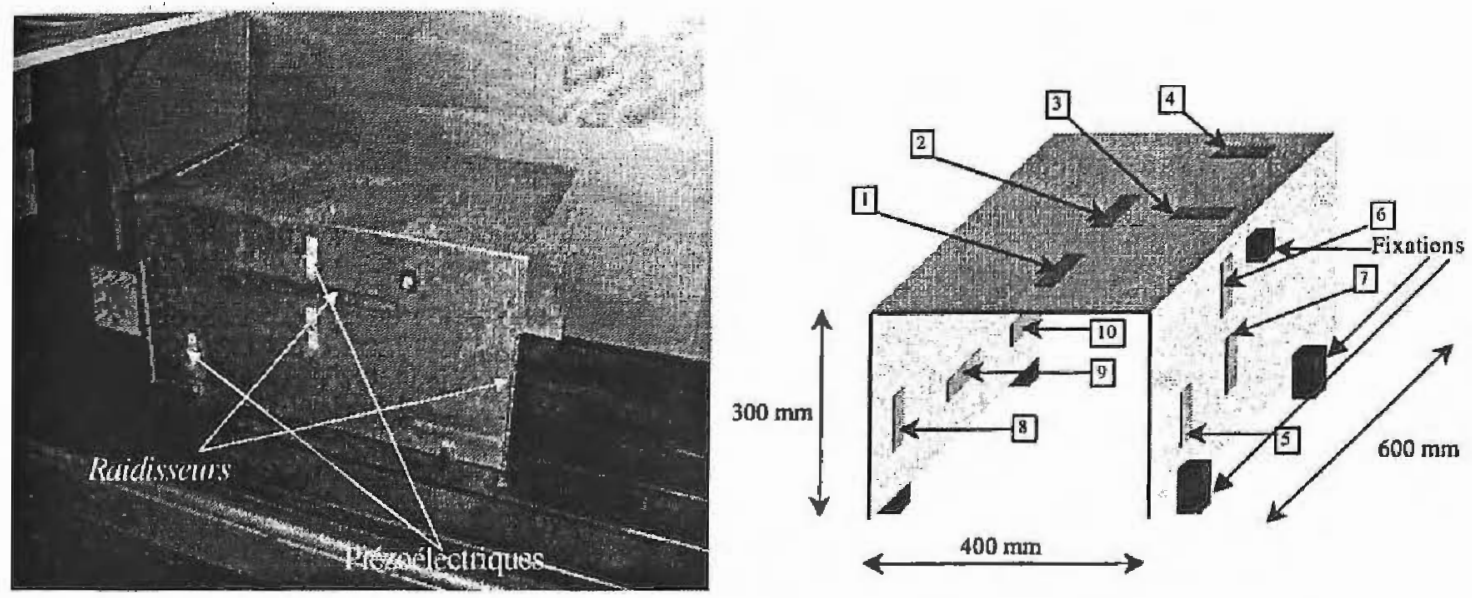

Figure 1. Structure CASYM

Cette structure est constituée d'une tôle en alliage d'aluminium pliée en U. Elle est fixée en 5 points sur un marbre (figure 1). Son épaisseur de $0,5 \mathrm{~mm}$ et ses dimensions conduisent à une structure très souple. Plusieurs raidisseurs sont fixés sur cette structure. Ces raidisseurs modifient les modes vibratoires et rendent la modélisation par éléments finis nettement plus complexe. Les actionneurs et capteurs sont constitués de 10 paires de céramiques piézo-électriques.

Cette maquette est complétée par une boucle de commande : des amplificateurs de charge pour le conditionnement des signaux de mesure, des amplificateurs de 
tension pour les actionneurs et une carte dSPACEC permettant l'implantation du régulateur. Le dispositif est décrit à la figure 2.

La figure 2 montre aussi que les céramiques piézo-électriques sont collées de part et d'autre de l'épaisseur de la tôle, idéalement en vis-à-vis exact. En pratique, les colles nécessitent un passage au four à une température voisine de $150^{\circ}$, ce qui provoque de légers glissements des piézos et des déformations locales de la tôle. Les faces extérieures des piézos sont reliées et sont donc au même potentiel. En revanche, la face interne est au potentiel de la structure elle-même reliée à la masse. Ce câblage impose des champs électriques de sens opposés dans les deux céramiques ce qui conduit à des sollicitations contraires : lorsque l'une se contracte, l'autre se dilate. La combinaison provoque l'apparition d'un moment de flexion pour les actionneurs. De façon similaire, seules les sollicitations de flexion, c'est-àdire les courbures, sont mesurées par ces capteurs.

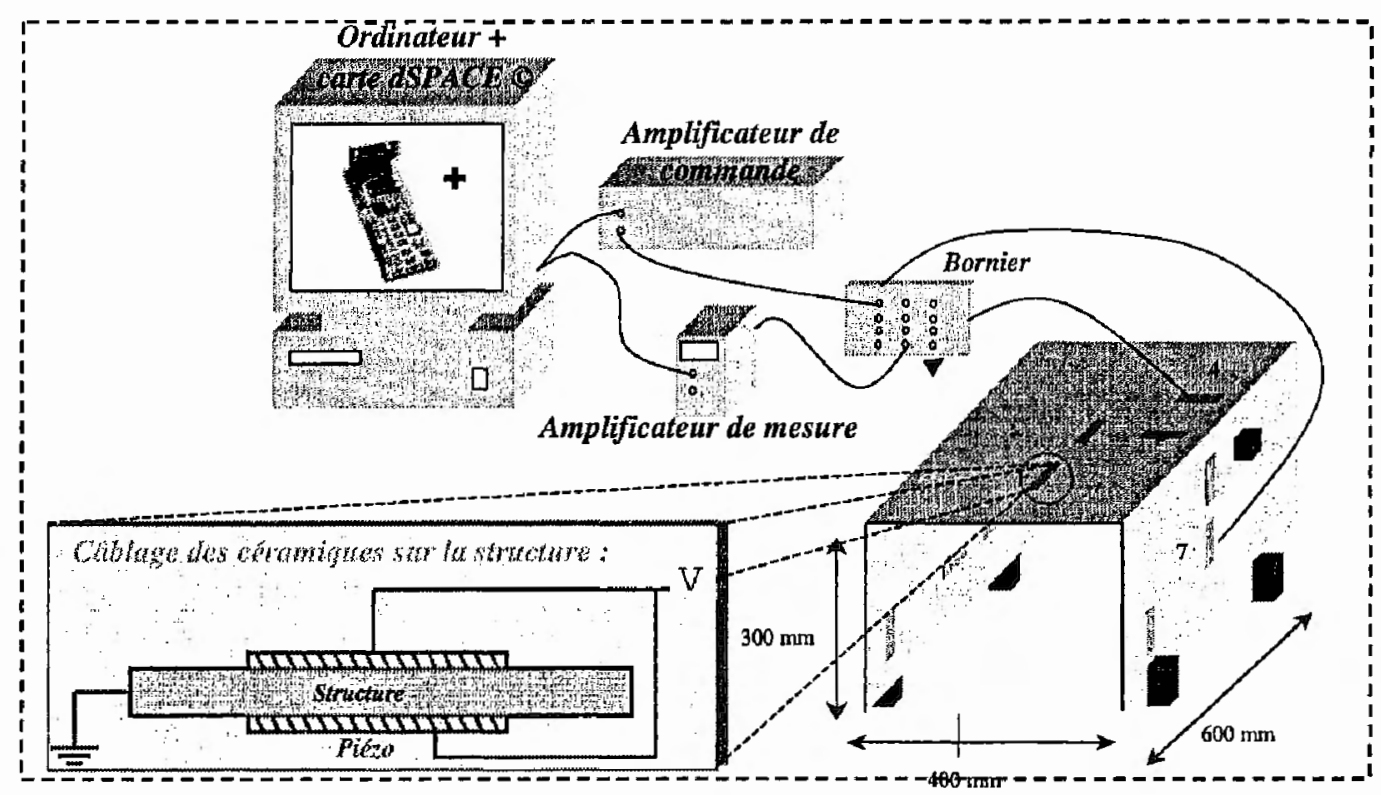

Figure 2. Dispositif et montage des céramiques

\subsection{Modélisation par éléments finis}

La modélisation par éléments finis suppose une définition correcte des parties mécaniques, ainsi que de celle des céramiques piézo-électriques. La flexibilité de la structure mécanique retenue conduit à un comportement vibratoire comportant de très nombreux modes en basses fréquences. Il s'ensuit que le recalage paramétrique est lourd et que les simulations sont relativement longues (plusieurs jours).

De façon générale, la méthode des éléments finis conduit à l'écriture de l'équation de mouvement de la structure mécanique sous la forme suivante : 


$$
M \underline{\ddot{q}}+C \underline{\dot{q}}+K \underline{q}=\underline{Q}
$$

où $M, C$ et $K$ sont respectivement les matrices masse, amortissement et raideur de la structure mécanique seule. $\underline{Q}$ est le vecteur des efforts généralisés. Le vecteur $\underline{q}$ représente le vecteur des déplacements nodaux. Ce dernier vecteur est généralement d'ordre très élevé noté nddl (nombre de degré de liberté).

Nous allons introduire dans ce modèle les éléments actifs de façon à prendre en compte leur influence sur le comportement dynamique de la structure et avoir accès aux grandeurs électriques (tensions de commande et mesure). Nous pourrons ainsi utiliser la ríchesse du modèle éléments finis pour effectuer les simulations préliminaires, nécessaires pour définir l'emplacement «optimal » et la «forme optimale » des capteurs et actionneurs (voir [BRU 99] pour plus de détails)

\subsubsection{Modélisation du couplage piézo-électrique}

L'effet piézo-électrique direct est le phénomène de polarisation électrique induite par l'application d'une contrainte à certains matériaux. Cette polarisation est proportionnelle à la contrainte et change de signe avec elle. Ce phénomène est réversible : une polarisation électrique résultant de l'application d'un champ électrique entraînera une déformation du même matériau. On parle alors d'effet piézo-électrique inverse.

L'origine de la piézo-électricité est liée à la structure cristalline du matériau : le phénomène ne s'observe que dans certains solides non conducteurs possédant une anisotropie cristalline caractéristique. Très peu de solides sont naturellement piézoélectriques, le quartz étant le plus connu. Les céramiques piézo-électriques de synthèse apparaissent dans les années quarante. Parmi ces matériaux de synthèse, les titanozirconates de plomb (PZT) sont les plus répandus.

Les relations de comportement suivantes sont obtenues à partir des deux premiers principes de la thermodynamique et en considérant un potentiel thermodynamique quadratique afin de décrire un comportement linéaire :

$$
\begin{array}{ll}
\text { Effet direct } & \underline{D}=\underline{\underline{e}} \varepsilon+\underline{\underline{\varepsilon}}^{\varepsilon} \underline{E} \\
\text { Effet inverse }: & \sigma=\underline{\underline{C}}^{E} \varepsilon-\underline{\underline{e}}^{T} \underline{E}
\end{array}
$$

où $\underline{E}$ et $\underline{D}$ représentent les vecteurs champ et déplacement électriques, $\underline{\underline{d}}$ et $\underline{\underline{e}}$ sont les matrices des coefficients piézo-électriques, $\sigma$ et $\mathcal{E}$ sont les tenseurs de contraintes et déformations, $\underline{\underline{\varepsilon}}^{\sigma}$ et $\underline{\varepsilon}^{\varepsilon}$ sont les matrices des constantes diélectriques à contraintes et déformations constantes et $\underline{\underline{C}}^{E}$ et $\underline{\underline{S}}^{E}$ sont les matrices de comportement mécanique à champ électrique constant. Pour plus de détails voir [GER 95, MAU 92, BRU 99]. 


\subsubsection{Prise en compte dans le modèle éléments finis}

Des éléments de type plaque sont placés aux emplacements des céramiques piézo-électriques pour modéliser leur masse et leur rigidité. $\mathrm{La}$ relation de comportement des céramiques est introduite dans la formulation de l'élément, ce qui conduit, à l'écriture de $\underline{\underline{Z}}$ sous la forme suivante [HEN 00] :

$$
\underline{Q}=\underline{Q}_{e x t}+Q_{a} u(t)
$$

$\underline{Q}_{e x t}$ représente les efforts mécaniques et $Q_{a} \cdot \underline{u}$ les effets de l'actionneur, $u(t)$ étant la tension de commande. De façon duale, la loi de comportement caractérisant l'effet piézo-électrique direct permet d'écrire, la tension de sortie $y(t)$ en fonction du vecteur des inconnues nodales mécaniques :

$$
y(t)=Q_{c} \underline{q}(t)
$$

\subsection{Simulations avec NASTRAN}

Le modèle par éléments finis et a été réalisé avec le logiciel MSC/NASTRAN qui dispose de capacités de modélisation très reconnues en dynamique linéaire. Comme la plupart des logiciels du commerce, il ne propose pas encore d'élément plaque spécifique permettant de prendre en compte un couplage piézo-électrique. Nous avons cependant utilisé la capacité du logiciel à autoriser une vaste gamme de sollicitations thermiques, pour l'utiliser dans ce travail.

La démarche adoptée, bien que très naturelle au sein d'une approche par éléments finis, est à notre connaissance originale. Elle est décrite succinctement, dans le cas où les deux céramiques piézo-électriques, chacune étant recouverte sur ses faces inférieure et supérieure d'une fine couche métallique conductrice, sont disposées de façon symétrique par rapport au plan moyen de la plaque. La modélisation de l'actionneur piézo-électrique de type plaque mince sandwich (piézo-élastique-piézo) ainsi formé peut être effectuée à partir de trois éléments de plaque élastiques superposés et décalés, par rapport au plan moyen, pour les deux céramiques. Cela est réalisé en utilisant les fonctionnalités classiques d'un logiciel éléments finis, pour peu que ce dernier, comme MSC/NASTRAN/PATRAN, permette l'excitation par des températures connues, variables en fonction du temps ou de la fréquence.

Considérons donc la construction d'un élément fini de plaque piézo-électrique. En complément des fonctions de base habituelles (pour décrire les déplacements et rotations), il est nécessaire d'adjoindre, des fonctions de base pour décrire le potentiel-électrique dans l'épaisseur de la pastille céramique [BRU 99]. Lorsque cette épaisseur est faible, et pour l'étude des premiers modes propres de vibration, le 
choix d'une variation du champ affine dans l'épaisseur, et constante dans le plan moyen de l'élément plaque, est suffisant pour obtenir un modèle convenable. Si l'on note $u$ la différence de potentiel appliquée entre les faces externes de la céramique, seule la composante $E_{3}$ du champ électrique, perpendiculaire au plan moyen, est non nulle. De plus, elle est homogène dans la céramique et égale à $u / h, h$ étant l'épaisseur. Ainsi, avec des notations évidentes, [3] peut se réécrire dans la plaque céramique :

$\sigma=\sigma_{\text {meca }}+\sigma_{\text {elec }}$, avec $: \sigma_{\text {elec }}=-e^{T} E=-e^{T}(0,0,1)^{T} E_{3}=-1 / h e^{T}(0,0,1)^{T} u=-\Sigma_{e l e c} u$

La prise en compte de la dilatation thermique dans la formulation s'appuie sur une relation de comportement thermomécanique de la forme :

$$
\sigma=\sigma_{m e c a}+\sigma_{t h} \text { avec }: \quad \sigma_{t h}=-C\left(\alpha_{1}, \alpha_{2}, \alpha_{3}, \alpha_{4}, \alpha_{5}, \alpha_{6}\right)^{T} \Delta T=-\Sigma_{t h} \Delta T
$$

Pour un matériau anisotrope général les $\alpha_{i}$ sont les coefficients de dilatation et $\Delta \mathrm{T}$ est la variation de température par rapport à la température de référence.

Dans le cadre de nos hypothèses $\sigma_{\text {elec }}$ et $\sigma_{\text {th }}$ jouent un rôle parfaitement analogue, et le choix de $\Delta T=u$ et des 6 coefficients de dilatation $\alpha_{i}$ tels que $\Sigma_{\text {th }}=-\Sigma_{\text {ele }}$ autrement dit tels que $\left(\alpha_{1}, \quad \alpha_{2}, \alpha_{3}, \alpha_{4}, \alpha_{5}, \alpha_{6}\right)^{T}=1 / h e^{T}(0,0,1)^{T}$ permet immédiatement de simuler une excitation électrique correcte dans la céramique sans aucune modification dans le logiciel.

Le modèle éléments finis complet utilise 1530 éléments plaque quadrilatéraux (type QUA), ce qui conduit à 9486 degrés de liberté. II fournit les déformées modales, les fréquences propres ainsi que les réponses fréquentielles.

Nous donnons à la figure 3 la déformée modale obtenue avec NASTRAN/PATRAN pour le mode 12,09 Hz. Sur cette figure apparaissent les régions où la déformée est constante. Ces régions sont grisées, le niveau de gris indiqué sur l'échelle latérale correspond à une déformation relative.

Ces simulations permettent de définir par rapport aux différents modes les céramiques les plus sensibles dans le cas où ces modes seraient excités. En effet, nous pouvons constater que certains modes ne sont pas observables par chaque céramique disposée sur la structure.

\subsection{Représentation d'état et réduction d'ordre}

En considérant le modèle par éléments finis précédent et en prenant en compte une entrée perturbatrice $d$, l'équation du mouvement de la structure flexible active s'écrit :

$$
M \underline{\ddot{q}}+C \underline{\dot{q}}+K \underline{q}=\underline{Q}_{a} u+\underline{Q}_{d} d
$$


Où $\underline{Q}_{a}$ et $\underline{Q}_{d}$ sont des matrices qui traduisent l'effet des actionneurs et de la perturbation sur la structure.

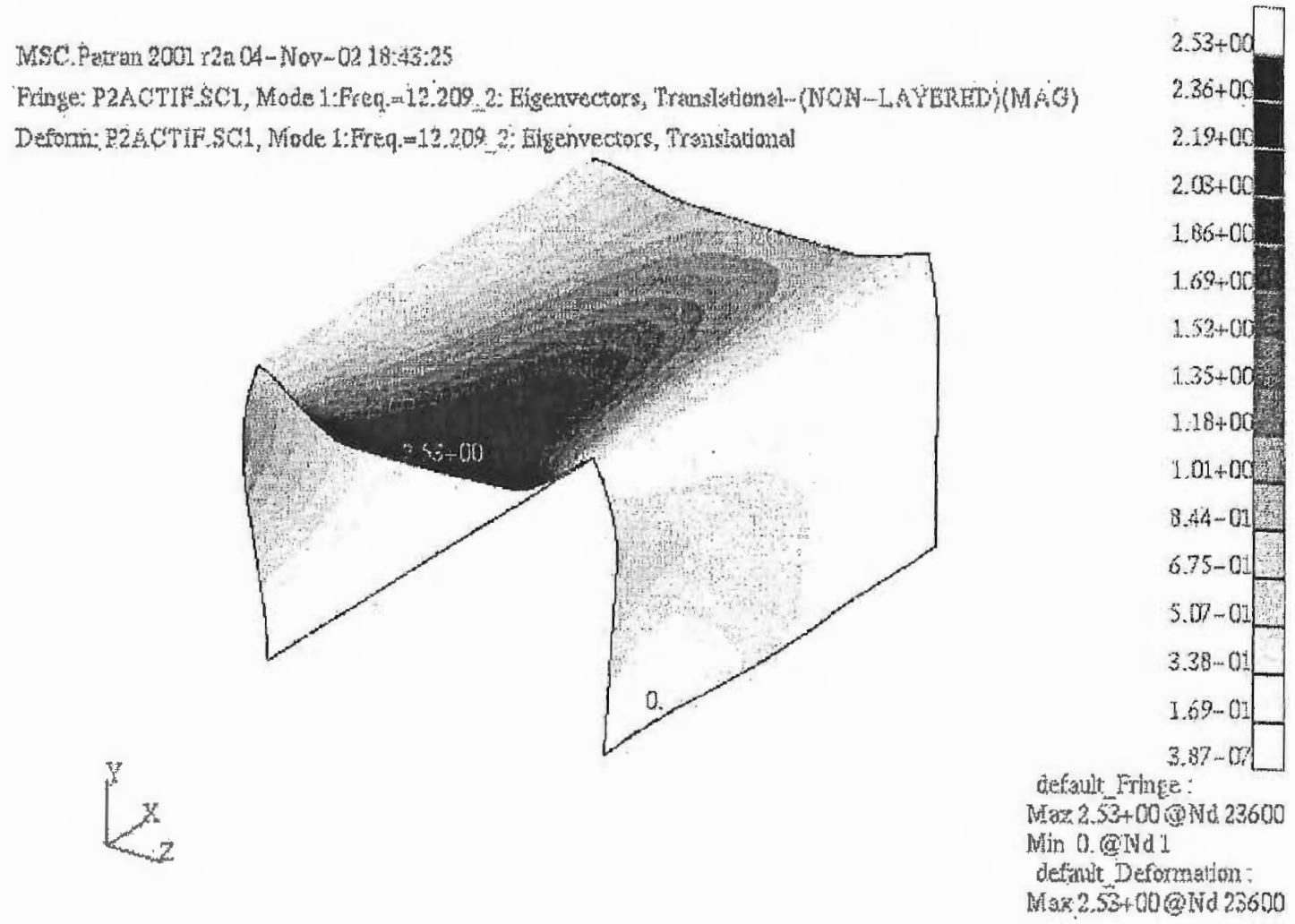

Figure 3. Déformée modale

Les modes propres du système sont obtenus par la résolution du problème généralisé aux valeurs propres :

$$
\left(K-\omega_{i}^{2} M\right) \underline{\phi}_{i}=\underline{0}
$$

avec $\omega_{i}$ et $\underline{\phi}_{i}$ les $i^{\text {eme }}$ pulsation et vecteur propre.

La résolution de [7] conduit à $n d d l$ pulsations et vecteurs propres. La matrice modale $\Phi$ est définie par :

$$
\Phi=\left[\begin{array}{llll}
\underline{\phi}_{1} & \underline{\phi}_{2} & \cdots & \underline{\phi}_{\text {nddl }}
\end{array}\right]
$$

Afin d'effectuer une commande de ce système, il est indispensable de réduire ce modèle. Cette réduction est basée sur la projection dans la base modale d'un nombre réduit de modes. Elle s'appuie sur la maximisation de la norme infinie du transfert entre les capteurs et actionneurs (voir [HEN 99]). Cette approche permet de définir une base modale tronquée $\Phi_{R}$ constituée de $n$ modes propres du système, dans laquelle nous projetons les déplacements nodaux pour obtenir le vecteur $\underline{g}$ des 
coordonnées généralisées. Le vecteur d'état $\underline{\omega} \cdot \underline{g}$ est formé en pondérant chaque composante du vecteur $\underline{g}$ par chaque pulsation propre $\omega_{i}$, soit :

$$
\underline{x}=\left[\left(\underline{\omega} \cdot \underline{g}^{T} \underline{\dot{g}}^{T}\right]^{T}\right.
$$

L'équation d'état est de la forme suivante (à une permutation d'état près) :

$$
\underline{\dot{x}}=\left[\begin{array}{cc}
0_{n} & \operatorname{diag}\left(\omega_{i}\right) \\
-\operatorname{diag}\left(\omega_{i}\right) & -2 \operatorname{diag}\left(\zeta_{i} \omega_{i}\right)
\end{array}\right] \underline{x}+\left[\begin{array}{c}
0_{n, 1} \\
\Phi_{R}^{T} \underline{Q}_{a}
\end{array}\right] u+\left[\begin{array}{c}
0_{n, 1} \\
\Phi_{R}^{T} \underline{Q}_{d}
\end{array}\right] d
$$

où les matrices diagonales sont de dimension $(n, n)$, et $\xi_{\mathrm{i}}$ représente l'amortissement associé à chaque mode. La dimension du vecteur d'état est alors $2 n$. L'équation de sortie est obtenue à partir de [5], soit :

$$
y=\left[Q_{c} \cdot \Phi_{R} \cdot \operatorname{diag}\left(1 / \omega_{i}\right) \quad 0_{1, n}\right] \underline{x}
$$

La représentation d'état ([10], [11]) est ensuite discrétisée en utilisant les techniques classiques [JAU 91] de passage de la représentation d'état continue en représentation d'état échantillonnée. La mise sous forme de transfert conduit à :

$$
y(k)=W\left(z^{-1}\right) u(k)+W_{d}\left(z^{-1}\right) d(k)
$$

où $W\left(z^{-1}\right)$ désigne la fonction de transfert du système à commander et $W_{d}\left(z^{-1}\right)$ celle de la perturbation exogène. $u(k)$ est le signal de commande.

\subsection{Identification de la structure}

Bien que les outils et les moyens de calculs actuels permettent de travailler sur des modèles de plus en plus riches, l'étape de modélisation constitue une simplification plus au moins grossière de la réalité physique. Pour conforter les hypothèses posées ou recaler les erreurs qu'elles ont engendrées, il est nécessaire de passer par la phase d'identification expérimentale du processus physique.

Nous avons ainsi identifié la structure CASYM en élaborant un premier modèle fondé sur les relevés expérimentaux des diagrammes de Bode et un deuxième modèle obtenu par l'algorithme récursif des moindres carrés.

\subsubsection{Relevés expérimentaux et premier modèle}

La structure étant complètement instrumentée, nous pouvons ainsi obtenir les courbes de Bode expérimentales pour chaque transfert entre un capteur et un 
actionneur. Nous qualifierons chaque couple capteurs-actionneurs de chemin. Ainsi, le chemin 3-4 représente l'actionneur 3 et le capteur 4.

En balayant un grand nombre de fréquences (entre $0-200 \mathrm{rad} / \mathrm{s}$ ), nous reconstituons un diagramme de Bode «expérimental» représentatif du comportement de la structure. Nous présentons (figure 4) les courbes de gain dans le plan de Bode relatives aux chemins $3-4$ et $8-4$ :
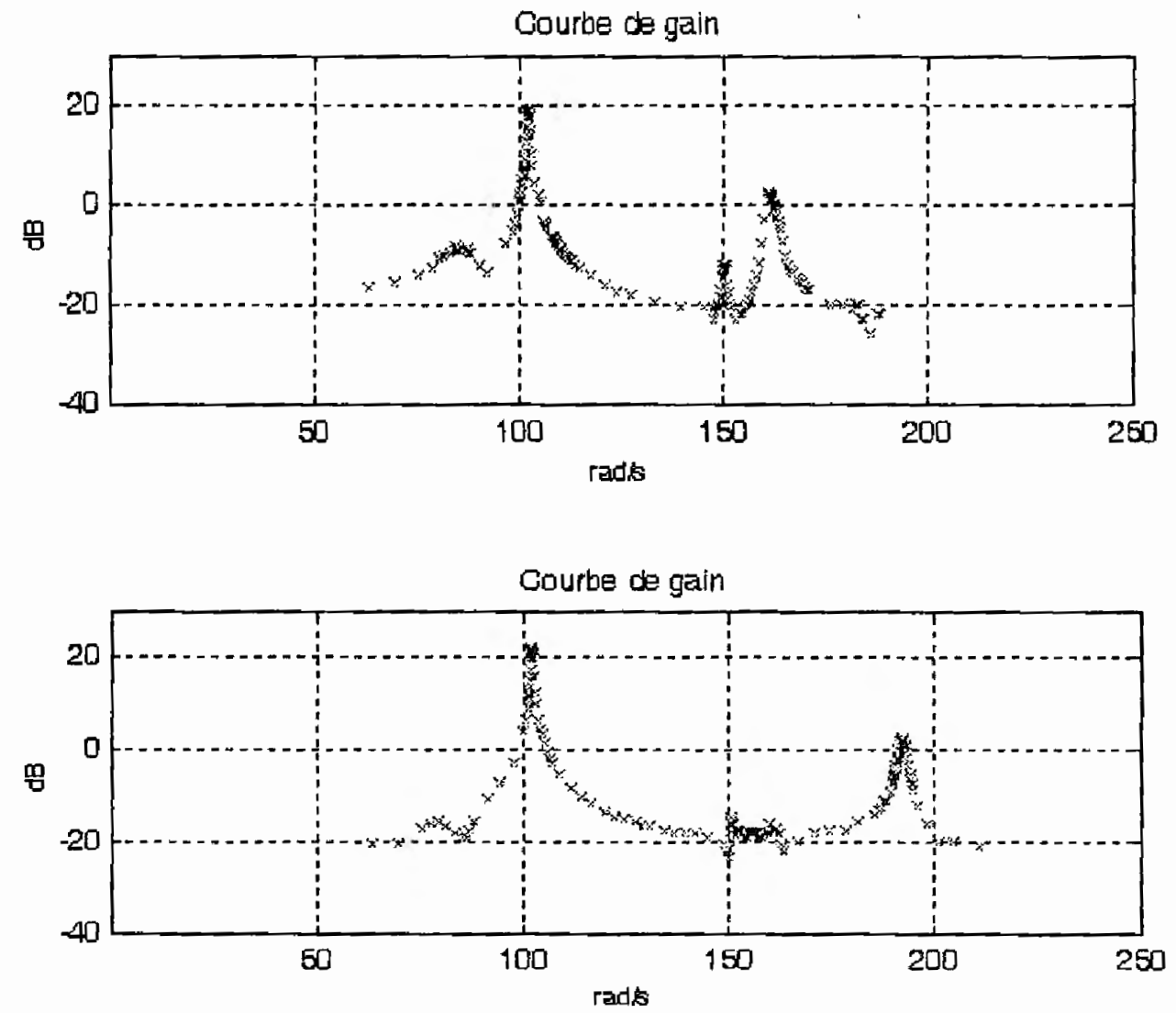

Figure 4. Courbes de bodes expérimentales : chemin 3-4 et chemin 8-4

Dans la plage de fréquence $0-200 \mathrm{rad} / \mathrm{s}$, chaque chemin possède 4 pics de résonance. Ces pics ou modes ne sont pas forcement les mêmes d'un chemin à un autre. En effet, ceci rejoint les simulations des déformées modales obtenues par NASTRAN (voir figure 3). Cependant, il faut garder à l'esprit que d'un point de vue mécanique, tous les modes sont présents sur la structure, mais, selon la disposition des céramiques, leur contribution sur les mesures est plus ou moins négligeable.

Pour élaborer un premier modèle, nous partons du fait que chaque pic correspond à un ensemble de deux pôles complexes conjugués. Il s'ensuit, qu'on peut représenter chacun de ces pics par un système du second ordre. Notre modèle sera alors constitue, pour chacun des chemins, de quatre systèmes du second ordre mis en parallèle. L'assemblage de ces fonctions dế transfert donne une nouvelle 
fonction de transfert continue constitué de 12 paramètres. En effectuant des simulations, et en ajustant les paramètres, nous avons déterminé un ensemble de paramètres permettant d'approcher au mieux les courbes du système réel et celles du système simulé. A la figure 5, nous comparons le chemin 3-4 (à gauche) et le chemin 8-4 (à droite).
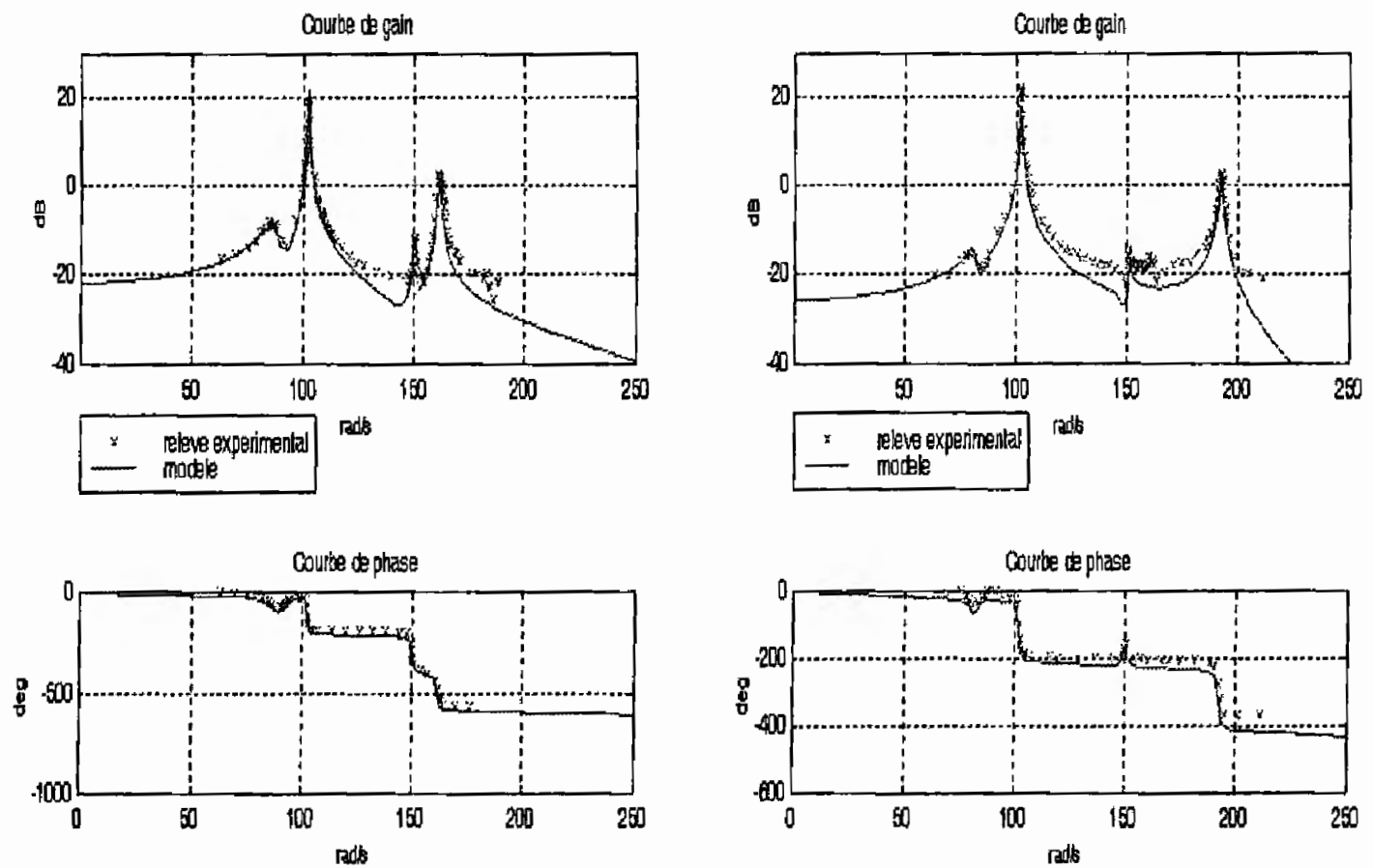

Figure 5. Diagrammes de Bode : expérimentations et modeles

De cette identification, nous observons que la résonance la plus importante se produit pour $\omega_{n}=102 \mathrm{rad} / \mathrm{s}$ et que les facteurs d'amortissement associés aux pics de résonance sont très faibles, de l'ordre de quelques millièmes.

\subsubsection{Identification récursive par moindres carrés}

La disponibilité d'un calculateur numérique permet de mettre en place des algorithmes d'estimation automatique des paramètres des modèles échantillonnés. La méthode récursive des moindres carrés (MCR), est la méthode d'identification la plus couramment utilisée ([LJU 87]). Elle doit en partie sa popularité à son utilisation très courante dans les algorithmes de commande adaptative.

La programmation de cet algorithme à été réalisé à l'aide du logiciel Simulink. Le progiciel ainsi élaboré possède l'avantage d'être directement exécutable en ligne par l'intermédiaire de la carte d'acquisition temps réelle dSPACEO.

Pour améliorer l'identification, nous filtrons l'entrée et la sortie par 2 filtres passe-bande Butterworth d'ordre 8 en parallèle afin à encadrer les pics de 
résonances. L'entrée est un bruit blanc et la période d'échantillonnage est fixée à $2 \mathrm{~ms}$.

Par exemple pour le chemin 3-4, nous obtenons la courbe de gain de la figure 6 :

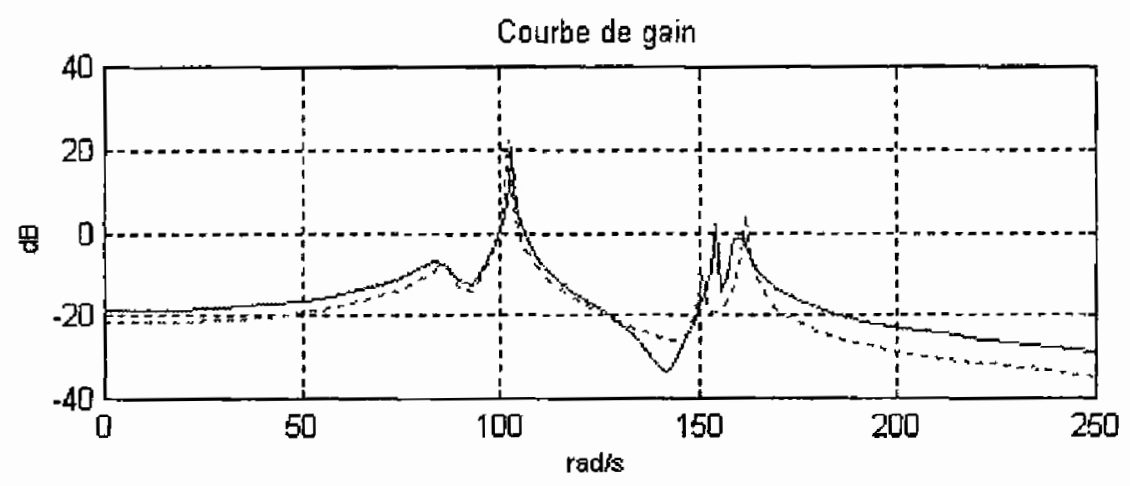

Figure 6. Identification MCR (trait plein) et premier modèle

Cette approche nous a donc permis de valider le premier modèle analytique obtenu à partir des courbes de Bode expérimentales. Ce modèle fournit donc une approximation suffisante du système réel dans la bande passante envisagée.

\subsubsection{Recalage du modèle éléments finis}

Dans le contrôle actif, l'identification est envisagée de façon différente suivant qu'on se place du point de vue mécanicien ou du point de vue automaticien. En effet, en mécanique l'objectif de l'étape d'identification est de recaler les paramètres physiques ou géométriques du modèle éléments finis (EF), comme la masse volumique, le module de Young ou la position des éléments fixés à la structure. Elle donne aussi la possibilité de recaler globalement les coefficients des matrices du modèle. En automatique, l'objectif de l'étape d'identification est d'obtenir un jeu de paramètres du modèle qui conduisent à des courbes dans le domaine temporel ou fréquentiel suffisamment proches de celles relevées expérimentalement.

Pour notre application, en comparant la courbe de Bode du chemin 3-4 obtenue par le modèle EF avec celle obtenue par l'identification expérimentale, nous observons des différences notoires. En effet, la courbe EF présente certaines résonances et anti-résonances qui n'apparaissent pas dans la courbe expérimentale. Une étape de recalage du modèle EF est donc nécessaire.

Le recalage du modèle $E F$ peut être effectué par une analyse modale expérimentale. Mais cette approche est délicate à mettre en œuvre en pratique ( $c f$. [QUE 94, GAW 98]). Dans notre cas, nous avons choisi de recaler modèle EF par rapport au modèle identifié expérimentalement. Le recalage s'effectue en minimisant un critère caractérisant l'écart entre les mesures et les résultats du modèle. 


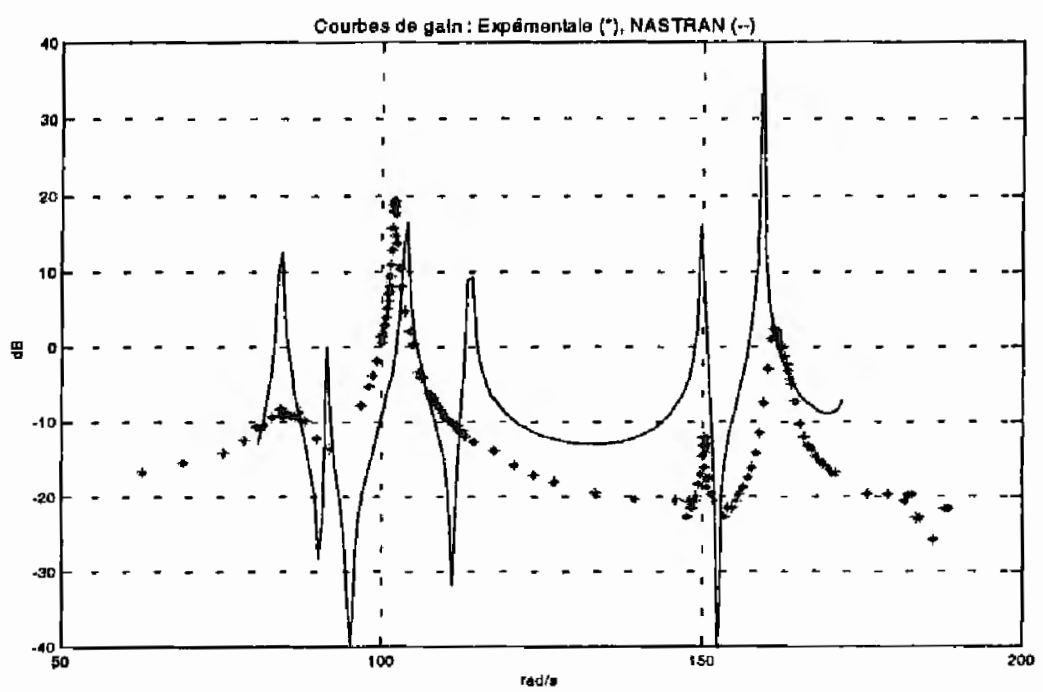

Figure 7. Courbes de Bode expérimentales et du modèle EF (trait plein)

\section{Commande adaptative par anticipation}

Dans cette étude, nous utilisons les actionneurs repérés 3 et 8 , et les capteurs repérés 4 et 5 . La perturbation (unique) est créée par la paire piézo-électrique 7 . La période d'échantillonnage est fixée à $2 \mathrm{~ms}$.

\subsection{Méthode}

Le filtrage adaptatif fait partie des régulateurs dénommés feedforward: on travaille par anticipation à partir de la mesure du signal de la perturbation.

Dans cette présentation, nous utilisons les filtres FIR (Finite Impulse Response), dont la fonction de transfert est notée :

$$
w(z)=w_{1} z^{-1}+w_{2} z^{-2}+\ldots .+w_{N} z^{-N}
$$

Le filtre FIR fait uniquement intervenir les signaux d'entrée (passés et présents). Ce filtre présente plusieurs avantages, parmi lesquels :

- il est toujours stable pour des valeurs finies de ses coefficients,

- il est relativement insensible aux faibles perturbations de ses coefficients.

Cependant, un système possédant une réponse temporelle très faiblement amortie nécessite un nombre $N$ très élevé de coefficients (plusieurs centaines).

De par sa relative simplicité et son faible volume de calculs, l'algorithme adaptatif de type gradient LMS est un algorithme très répandu [WID 85]. L'algorithme filtered-xLMS en constitue une amélioration et fut d'abord présenté par 
Elliot et al. [ELL 87]. Il se distingue de l'algorithme LMS par le filtrage de la perturbation au travers d'une fonction de transfert représentant le transfert entre les entrées et les sorties de la structure mécanique (voir la figure 5). En nous inspirant de [KUO 96] et [ELL 87], cet algorithme est étenđu ici aux systèmes possédant plusieurs entrées et plusieurs sorties. Nous présentons cette méthode pour le système CASYM ayant 2 entrées et 2 sorties.

Le signal de commande est établi à partir du signal de perturbation par l'intermédiaire du filtre dont les coefficients sont mis à jour grâce aux signaux d'erreur (voir figure 8).

La figure 8 fait apparaitre la structure CASYM avec ses entrées ( $u_{3}$ et $u_{8}$ ), ses sorties $\left(y_{4}\right.$ et $y_{5}$ ) et le signal de perturbation $d_{7}$ connu. Les $P u_{i j}$ représentent les estimations des fonctions de transfert entre l'entrée $i$ et la sortie $j$. Algorithme filtered-x LMS dans le cas 2 entrées, 2 sorties :

Dans le schéma présenté figure 8 , les valeurs mesurées de l'erreur de sortie sont rangées dans le vecteur colonne:

$$
y(k)=\left[y_{4}(k) \quad y_{5}(k)\right]^{\mathrm{T}}
$$

Les filtres de commande $\underline{w}_{3}$ et $\underline{w}_{8}$, chacun d'ordre $N$, agissent sur les 2 actionneurs d'entrée.

$$
\underline{w}_{p}(k)=\left[w_{p, 1}(k) \quad w_{p, 2}(k) \quad \cdots \quad w_{p, N}(k)\right]^{T} \quad p=3,8
$$

L'ensemble des vecteurs de coefficients $\underline{w}_{p}(k)$ des 2 filtres de commande est regroupé dans le vecteur colonne $W(k)$ de dimension $2 \mathrm{~N}$ :

$$
W(k)=\left[\underline{w}_{3}^{T}(k) \quad \underline{w}_{8}^{T}(k)\right]^{T}
$$

Les sorties des filtres de commande sont les entrées de CASYM, elles sont notées :

$$
\underline{u}(k)=\left[\begin{array}{ll}
u_{3}(k) & u_{3}(k)
\end{array}\right]^{T}
$$

La $p^{\text {ème }}$ composante, notée $u_{p}(k)$ est déterminée en filtrant la perturbation à travers le filtre FIR correspondant $\underline{w}_{p}(k)$ :

$$
u_{p}(k)=\underline{w}_{p}^{T}(k) \cdot \underline{d}(k) \quad p=4,5
$$

où les valeurs du signal de perturbation sont regroupées dans le vecteur :

$$
\underline{d}(k)=\left[\begin{array}{llll}
d(k) & d(k-1) & \cdots & d(k-N+1)
\end{array}\right]^{T}
$$


Le vecteur constitué des échantillons du signal de perturbation aux instants passés et présents est placé dans une matrice $2 \mathrm{~N} \times 2$ :

$$
D(k)=\left[\begin{array}{cc}
\underline{d}(k) & 0 \\
0 & \underline{d}(k)
\end{array}\right]
$$

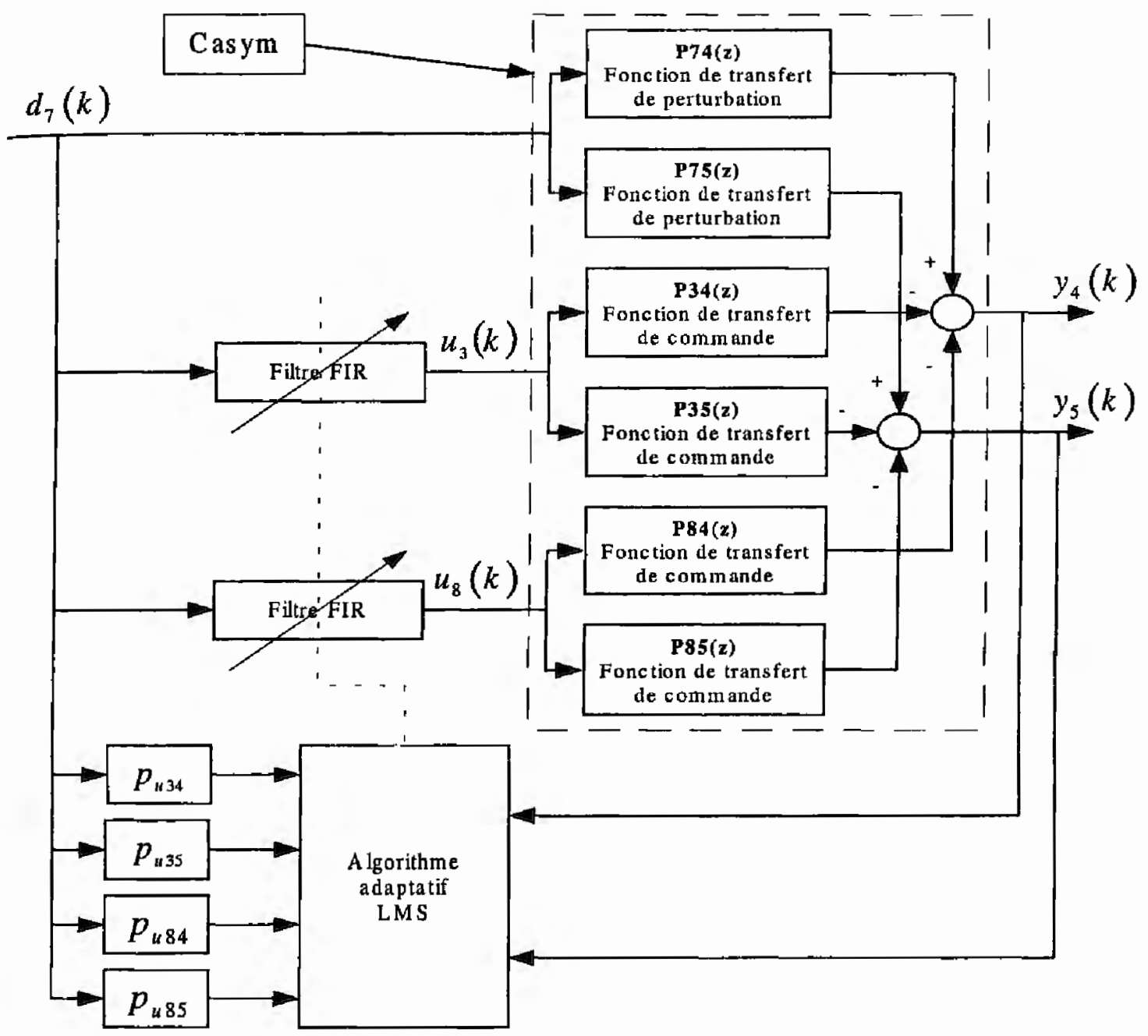

Figure 8. Schéma de principe

Ainsi les sorties des filtres FIR sont obtenues par :

$$
\underline{u}(k)=D^{T}(k) \cdot W(k)
$$

La réponse $\underline{y}_{u}(k)$ du système aux entrées $\underline{u}(k)$ est déterminée par la convolution :

$$
\underline{y}_{u}(k)=P_{u}(k) * \underline{u}(k)
$$


$P_{u}(k)$ est la matrice $2 N \times 2$ contenant les coefficients des réponses impulsionnelles discrètes (RID) :

$$
P_{u}(k)=\left[\begin{array}{ll}
\underline{p}_{u 34}(k) & \underline{p}_{u 84}(k) \\
\underline{p}_{u 35}(k) & \underline{p}_{u 85}(k)
\end{array}\right]
$$

Le vecteur d'erreur est exprimé par la différence entre le vecteur perturbation et le vecteur représentant l'influence des entrées sur la structure CASYM (voir figure 8):

$$
\underline{y}(k)=\underline{y}_{r}(k)-\underline{y}_{u}(k)
$$

En substituant [22] dans [24], on obtient :

$$
\underline{y}(k)=\underline{y}_{r}(k)-P_{u}(k) * \underline{u}(k)
$$

ou encore en tenant compte de [21] on a :

$$
\underline{y}(k)=\underline{y}_{r}(k)-P_{u}(k) *\left[D^{T}(k) \cdot W(k)\right]
$$

Pour réaliser le mécanisme d'adaptation des coefficients du filtre, nous définissons la fonction coût suivante :

$$
\xi=\sum_{m=1}^{2} y_{m}^{2}(k)
$$

La minimisation de ce critère conduit à la mise à jour des coefficients du filtre FIR dans la direction inverse du gradient instantané :

$$
W(k+1)=W(k)-\frac{\mu}{2} \cdot \hat{\nabla}(\xi(k))
$$

Le gradient est estimé par :

$$
\hat{\nabla}(\xi(k))=-2 \cdot\left[\begin{array}{ll}
\hat{p}_{u 34}^{T} \underline{d}(k) & \hat{\hat{p}}_{u 33}^{T} \underline{d}(k) \\
\underline{\hat{p}}_{u 84}^{T} \underline{d}(k) & \underline{\hat{p}}_{u 85}^{T} \underline{d}(k)
\end{array}\right] \cdot \underline{y(k)}
$$

où les termes $\underline{\underline{p}}_{u i j}$ sont les estimations de la RID entre le $i^{\text {ème }}$ actionneur et le $j^{\text {ème }}$ capteur. Chaque terme de la matrice $P$ peut être considéré comme la convolution entre $\underline{\hat{p}}_{u i j}$ et $\underline{d}(k)$. Notons $\underline{\hat{d}}_{u i j}$ le signal de perturbation filtré par $\hat{p}_{u i j}$ : 


$$
\underline{\hat{d}}_{u l j}=\underline{\hat{p}}_{u i j} * \underline{d}(k)
$$

Définissons alors la matrice :

$$
\hat{d}_{u}=-2\left[\begin{array}{ll}
\hat{d}_{u 34}(k) & \hat{\hat{d}}_{u 35}(k) \\
\underline{\hat{d}}_{u 84}(k) & \underline{\hat{d}}_{u 85}(k)
\end{array}\right]
$$

Ainsi, le gradient [29] s'écrit :

$$
\hat{\nabla}(\xi(k))=-2\left[\begin{array}{ll}
\hat{\hat{d}}_{u 34}(k) & \hat{\hat{d}}_{u 35}(k) \\
\underline{\hat{d}}_{u 84}(k) & \underline{\hat{d}}_{u 85}(k)
\end{array}\right] \cdot y(k)
$$

La combinaison de [28] et [31] donne l'algorithme nommé LMS filtered-x [ERI 92] en version 2 entrées, 2 sorties :

$$
W(k+1)=W(k)+\mu \cdot \hat{d}_{u}(k) \cdot y(k)
$$

Comme indiqué par [33], la mise à jour des coefficients du filtre est obtenue en ajoutant aux coefficients actuels du filtre $W(k)$ le produit de la perturbation filtrée $\hat{d}_{u}$ par le vecteur d'erreur de sortie $\underline{y}(k)$. Il faut remarquer que cette méthode suppose une connaissance précise des fonctions de transfert. Pour des raisons numériques, et en supposant une identification correcte de ces transferts $\hat{d}_{u}$, il est plus efficace de les représenter par une fonction de transfert en $z$. Dans ces conditions, le volume de calculs est fortement diminué.

\subsection{Simulations}

Dans cette simulation, la perturbation est un signal sinusoïdal à la pulsation fixe de $102 \mathrm{rad} / \mathrm{s}$ et d'amplitude $100 \mathrm{mV}$. La figure 9 présente les signaux $y_{4}$ et $y_{5}$ en régime stabilisé. Le système est en boucle ouverte pendant la première seconde, puis il passe en boucle fermée jusqu'à l'instant $20 \mathrm{~s}$. 

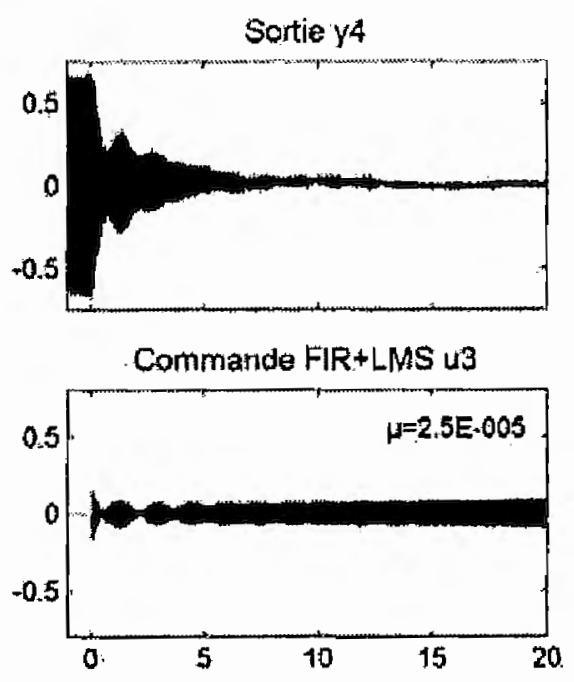
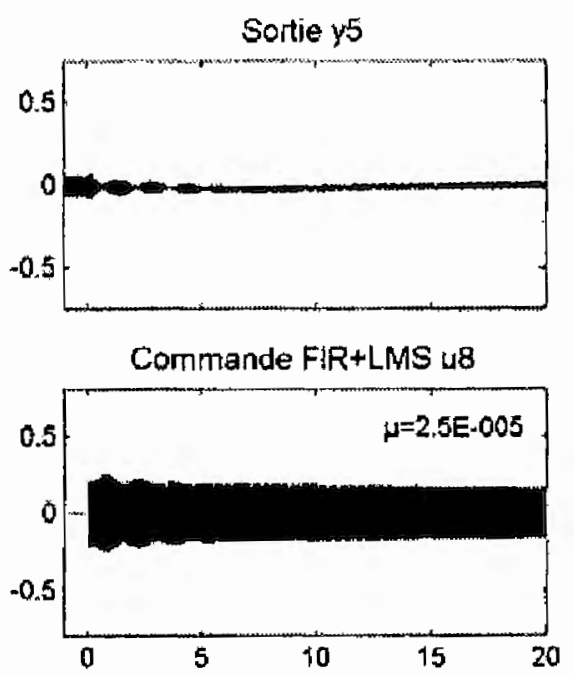

Figure 9. Perturbation à fréquence fixe

REMARQue. - C'est le capteur $y_{4}$ qui est le plus sensible. Le régulateur combat la perturbation avec un temps de réponse de $15 s$ environ.

\section{Commande robuste par boucle sur les sorties}

\subsection{Méthode}

La commande $H_{\infty}$ est attractive à cause de ses propriétés de robustesse [SKO 96]. Cette commande repose sur la minimisation de la norme infinie d'une fonction de transfert particulière. Pour effectuer la synthèse du correcteur, nous considérons que le système est continu. La commande $H_{\infty}$ ne nécessite pas la mesure de la perturbation $d(t)$, ce qui constitue un intérêt évident. Elle ne nécessite que la connaissance des mesures $y(t)$. Nous avons vu, lors de la modélisation, que nous avons négligé de nombreux modes. Les fréquences de résonances conservées sont supposées parfaitement connues et invariables (pas d'incertitude paramétrique). Il faut donc, prendre en compte les modes négligés afin de réaliser un correcteur robuste. Pour cela, définissons l'incertitude multiplicative non structurée $\Delta(\mathrm{p})$ par :

$$
\Delta(p)=G_{r}(p) \cdot G(p)^{-1}-I
$$

où : $G_{r}(p)$ est la matrice de transfert réelle (ordre 30) et, $G(p)$ est la matrice de transfert du modèle nominal (ordre 18). 
Pour déterminer le correcteur $K(p)$ il faut choisir les matrices de pondération $W 1(p), W 2(p)$ et $W 3(p)$ pondérant respectivement les vecteurs d'erreur $\underline{e}$, le vecteur d'entrée $\underline{u}$ et le vecteur de sortie $\underline{x}$ ( $c f$. figure 10).

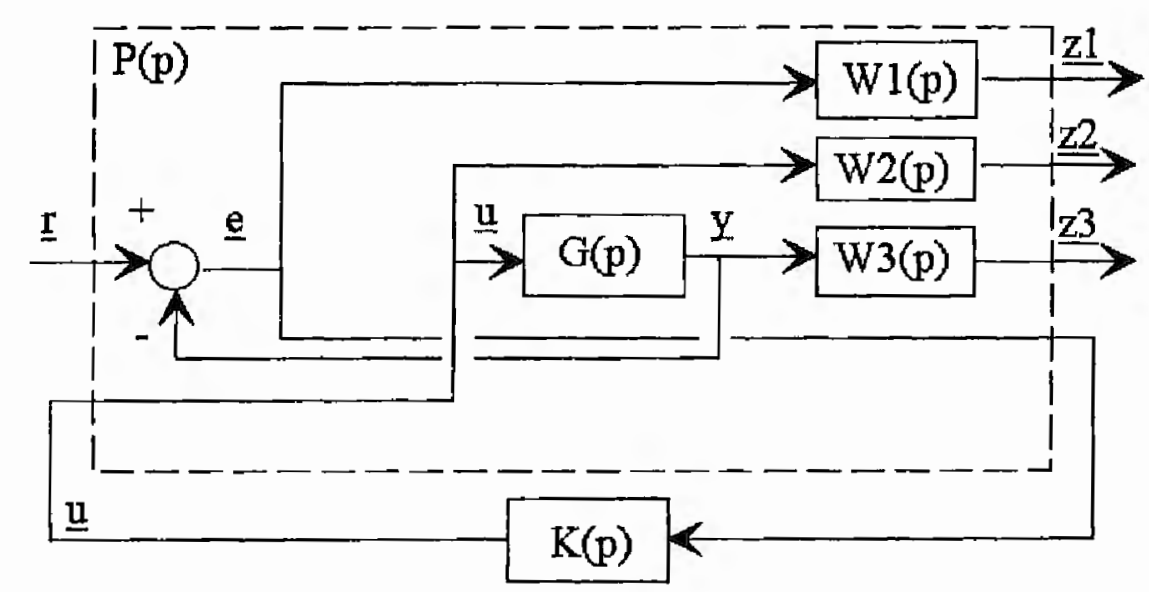

Figure 10. Représentation standard

Le principe de la synthèse des correcteurs $K(p)$ consiste à rechercher un correcteur qui minimise (problème de sensibilité mixte) la norme suivante :

$$
\left\|\begin{array}{c}
W 1 . S \\
W 2 . K . S \\
W 3 . T
\end{array}\right\|_{\infty}
$$

où la sensibilité $S(p)$ et la sensibilité complémentaire $T(p)$ sont données par :

$$
\begin{aligned}
& S=(I+G \cdot K)^{-1} \\
& T=G \cdot K \cdot(I+G \cdot K)^{-1}
\end{aligned}
$$

- La matrice $W 3(p)$ détermine la robustesse du système bouclé. Nous l'avons choisie de degré réduit et majorant l'incertitude multiplicative. La matrice W3 est ici une matrice carrée 2,2 où chaque terme est une fonction de transfert dont les numérateurs et dénominateurs sont de degré 2 . Les valeurs numériques de ces transferts s'obtiennent en traçant les courbes de Bode de $\Delta(p)$ et en choisissant $W 3(p)$ afin que les courbes de Bode des gains de $W 3(p)$ majorent les gains de $\Delta(p)$. Il faut remarquer que la matrice $W 3(p)$ n'est pas diagonale.

- La matrice $W 1(p)$ est une matrice $2 \times 2$ choisie de la forme : 


$$
W 1(p)=\left[\begin{array}{cc}
\left(\frac{g_{1} \cdot \omega_{1}^{2}}{p^{2}+2 \cdot \xi_{1} \cdot \omega_{1}+\omega_{1}^{2}}\right)^{2} & 0 \\
0 & \left(\frac{g_{2} \cdot \omega_{2}^{2}}{p^{2}+2 \cdot \xi_{2} \cdot \omega_{2}+\omega_{2}^{2}}\right)^{2}
\end{array}\right]
$$

Pour obtenir un rejet maximum autour de la pulsation de résonance $\omega_{n}=102 \mathrm{rad} / \mathrm{s}$, nous avons retenu $\omega_{1}=\omega_{2}=102 \mathrm{rad} / \mathrm{s}$. Les facteurs d'amortissements $\xi_{1}$ et $\xi_{2}$ jouent sur l'amplitude des pics de résonance ainsi que sur les bandes passantes de ces pics. Pour rejeter efficacement une perturbation de pulsation $\omega_{1}$, nous avons intérêt à prendre $\xi_{1}$ et $\xi_{2}$ très faibles, mais pour rejeter une perturbation ayant la pulsations au voisinage de $\omega_{1}$ il est préférable d'augmenter le facteur d'amortissement $\xi_{1}$. Plusieurs simulations ont conduits à $\xi_{1}=\xi_{2}=0,02$.

Les gains statiques $g_{1}$ et $g_{2}$ sont choisis de telle façon à ce que, après calcul du correcteur, on vérifie les inégalités [39] et [40]. En pratique, nous commençons par des gains relativement élevés, nous calculons le correcteur, puis nous diminuons progressivement ces deux gains en vérifiant que :

$$
\begin{aligned}
& \bar{\sigma}(S(p))<\bar{\sigma}\left(W 1^{-1}(p)\right) \\
& \bar{\sigma}(T(p))<\bar{\sigma}\left(W 3^{-1}(p)\right)
\end{aligned}
$$

où $\bar{\sigma}(S(p))$ désigne la valeur singulière maximale de la matrice $S(p)$.

Après avoir calculé le correcteur en continu, $K(p)$, nous l'avons simplement échantillonné avec la période $T=0,002 s$. Pour réaliser l'implantation en temps réel, chaque bloc de transfert du correcteur est réduit en utilisant la méthode classique basée sur la comparaison des grammiens de gouvernabilité et d'observabilité. Après réduction, les dénominateurs de fonctions de transfert des correcteurs ont des degrés compris entre 8 et 12 .

\subsection{Simulations}

La figure 11 donne l'évolution de la sortie issue du capteur 4 en boucle fermée (courbe du haut) et en boucle ouverte (courbe du bas) lorsque la perturbation sinusoïdale à $\omega_{1}=102 \mathrm{rad} / \mathrm{s}$ est appliquée à partir de l'instant $t=0 \mathrm{~s}$.

La figure 12 montre l'efficacité du correcteur. Il faut cependant insister sur les conditions idéales de cette simulation : le choix de $W 1$ est adapté à la pulsation de la perturbation. Le choix de W3 repose sur la modélisation des matrices de transfert nominale et incertaine de la structure CASYM. 

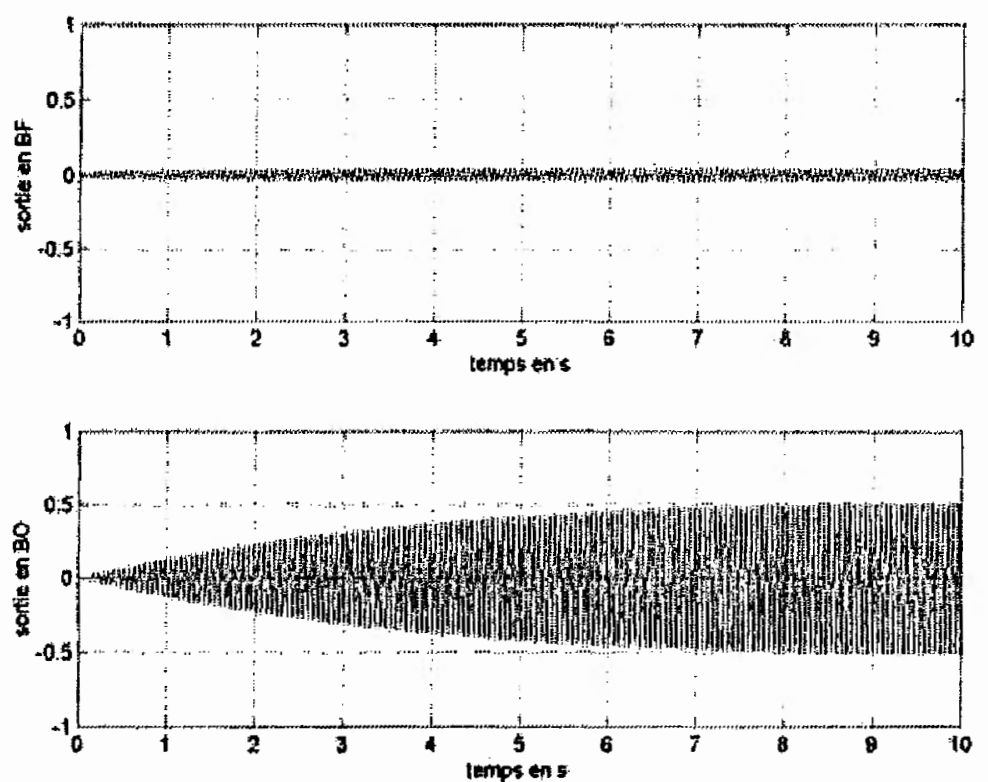

Figure 11. Rôle du correcteur $H_{\infty}$

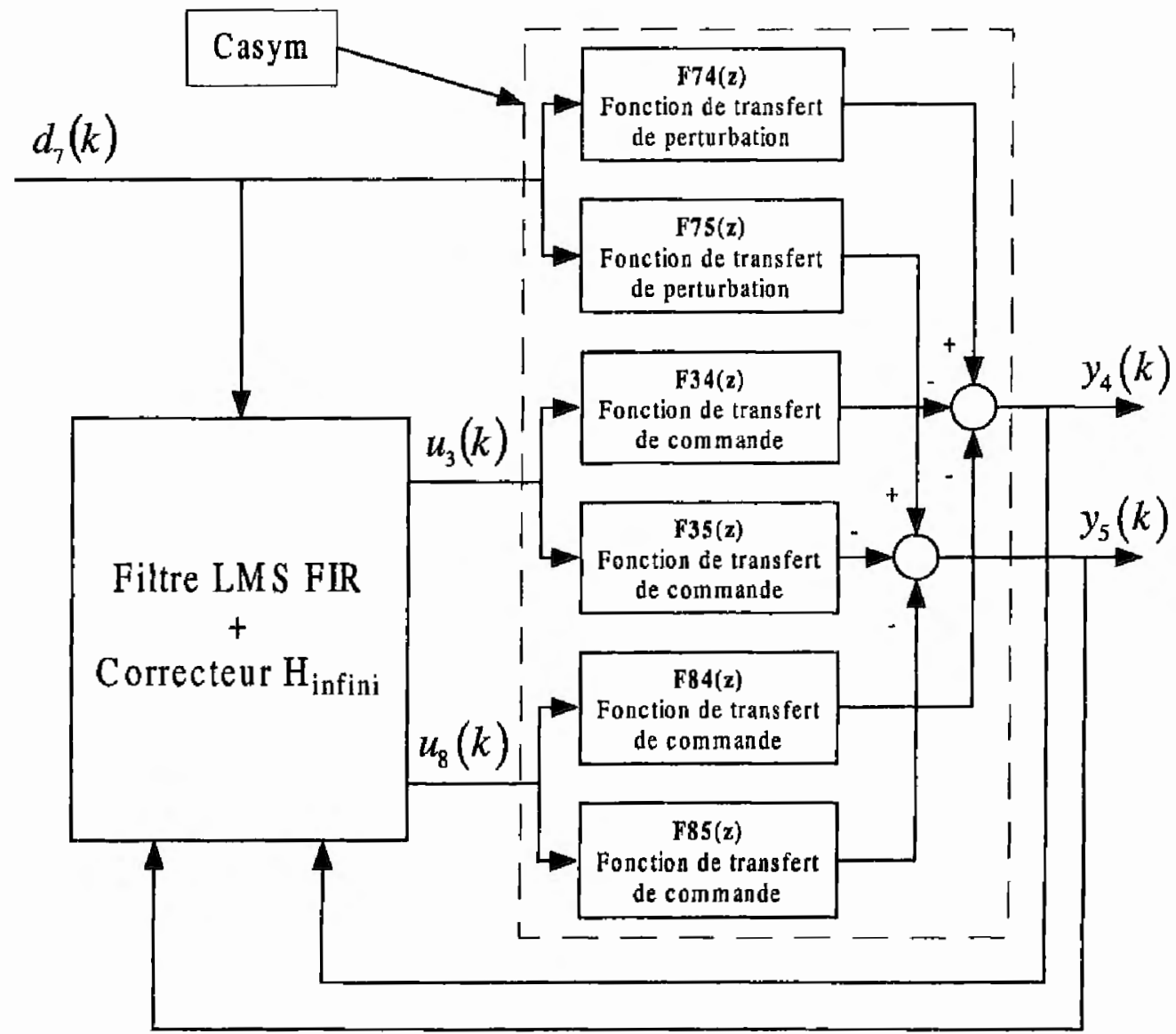

Figure 12. Commande mixte 


\section{Commande mixte}

\subsection{Principe}

La commande adaptative par anticipation fournit de très bons résultats dans le cadre d'une perturbation sinusoïdale lentement variable. La commande $H_{\infty}$ fournit des performances convenables pour des perturbations non mesurées. Cette commande stabilise rapidement le système perturbé. L'association des deux méthodes vise à rejeter les perturbations dans un contexte plus large que celui des perturbations sinusoïdales. Notons que la commande par anticipation ne modifie pas les pôles du système bouclé obtenu avec la commande $H_{\infty}$.

Le schéma de principe est donné à la figure 12. Les signaux de commande $u_{3}(k)$ et $u_{8}(k)$ sont simplement la somme des signaux calculés par le filtre adaptatif et de ceux calculés par la commande $H_{\infty}$.

\subsection{Expérimentations}

Lors des expérimentations nous avons noté une très bonne coïncidence entre les simulations qui ont permis la mise au point des régulateurs et les expérimentations sur la structure CASYM. Dans cette section, nous ne donnons que quelques résultats expérimentaux. Dans tous les essais, l'amplitude de la perturbation $\left(u_{7}\right)$ est fixée à $250 \mathrm{mV}$, cette perturbation apparaît à l'instant $2 \mathrm{~s}$.

Dans cet article, trois campagnes d'essais sont présentées :

- Le premier essai concerne une perturbation sinusoïdale fixe à $102 \mathrm{rad} / \mathrm{s}$, ce qui correspond à une résonance sur les courbes de Bode de la figure 5 . Nous présentons l'évolution temporelle des entrées et des sorties aux figures 13, 14 et 15 . A la figure 13 la commande $H_{\infty}$ est utilisée seule, à la figure 14, la commande adaptative est utilisée seule alors qu'à la figure 15 la commande mixte est utilisée.
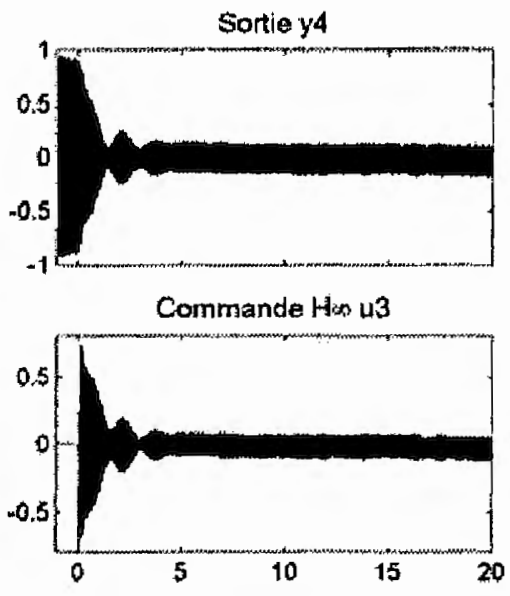
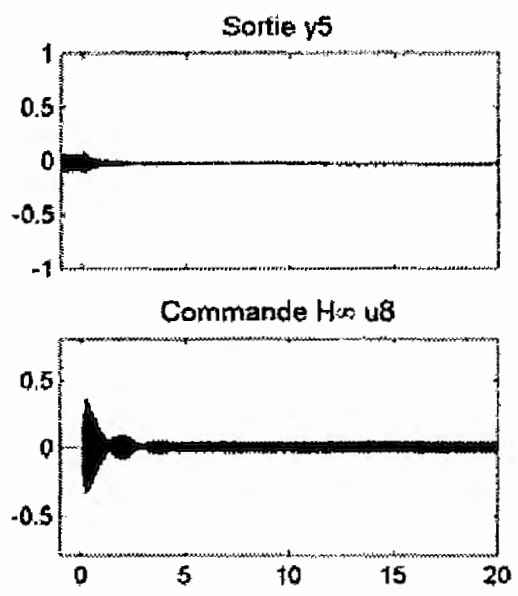

Figure 13. Expérimentation pour le correcteur $H_{\infty}$ seul 
La figure 13 montre que la commande $H_{\infty}$ rejette bien la perturbation. Ceci provient en grande partie du choix de la fonction de pondération $W 1(p)$ qui est bien adaptée à la nature de la perturbation. Cependant, on note que le capteur 4 reste sensible à cette perturbation, malgré une réelle atténuation de l'effet de la perturbation sur la sortie.
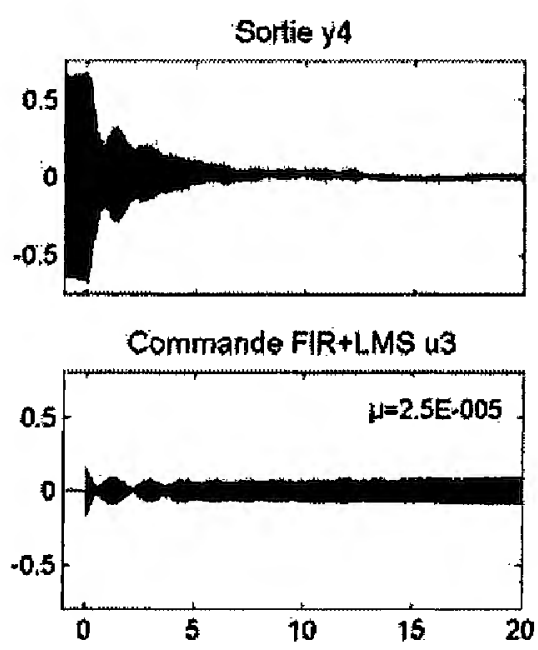
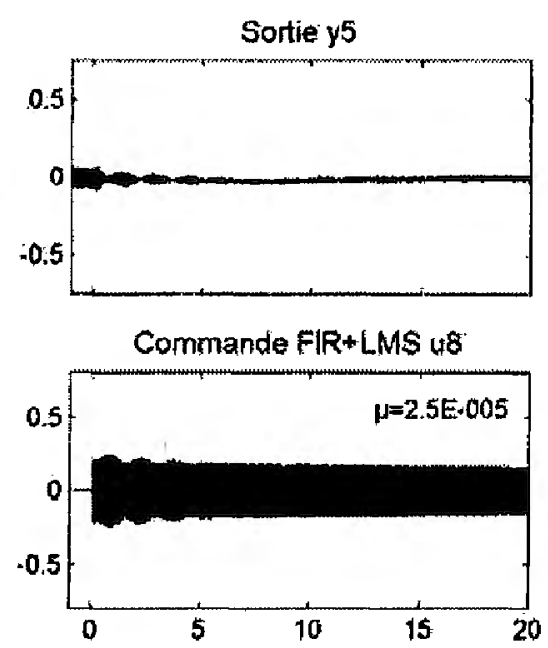

Figure 14. Expérimentation pour le filtre adaptatif FIR

REMARQUE. - La figure 14 fait apparaître un meilleur rejet de la perturbation au détriment d'un temps de réponse légèrement plus long.

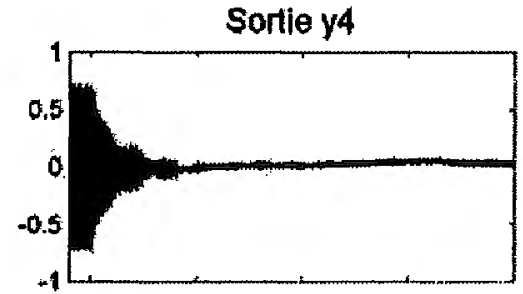

Commande $\mathrm{H}$ or u3

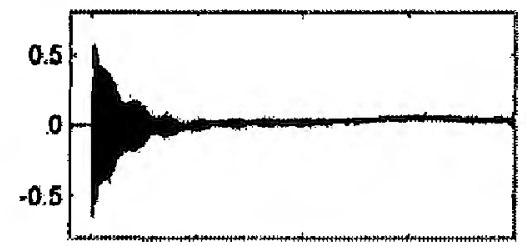

Commande FIR+LMS U3

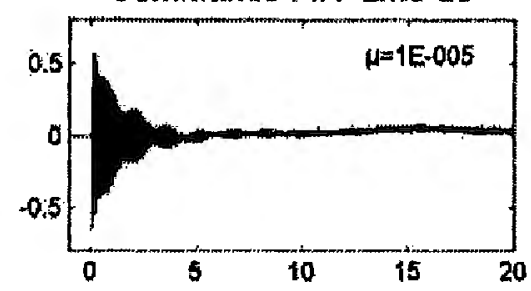

Sorlie y5

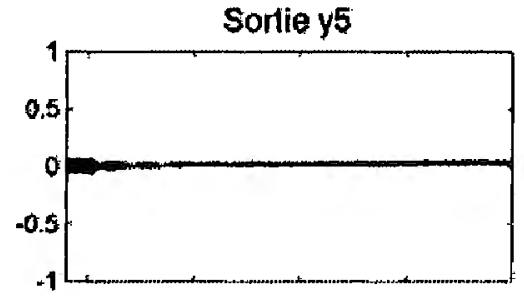

Commande Hoo 48

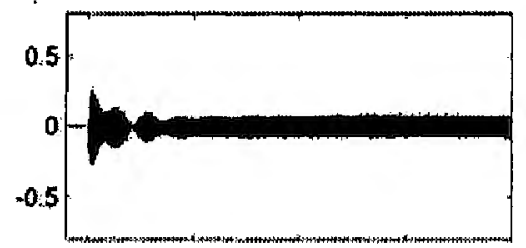

Commande FIR+LMS us

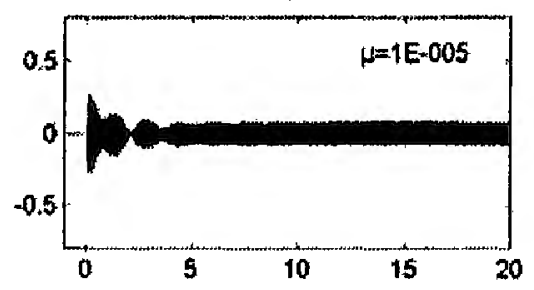

Figure 15. Expérimentation pour la méthode mixte 
REMARQUE.- La figure 15 présente de façon séparée les signaux de commande provenant respectivement du correcteur $H_{\infty}$ et de la commande adaptative. On note que le rejet de la perturbation est excellent et que les actionneurs sont d'avantage sollicités. La méthode mixte est ici la plus performante, à la fois en termes d'efficacité et de rapidité puisque le rejet est presque total en moins de $5 \mathrm{~s}$.

- Le deuxième essai concerne une perturbation sinusoïdale où la pulsation varie de $90 \mathrm{rad} / \mathrm{s}$ à $110 \mathrm{rad} / \mathrm{s}$ en $200 \mathrm{~s}$. (voir figure 16 ).

REMARQUES.- La commande mixte fournit des résultats meilleurs que ceux obtenus pour chaque commande utilisée seule. Cependant, il faut noter que la vitesse d'évolution de la perturbation ne doit pas être excessive car la mise à jour des coefficients du filtre s'effectue relativement lentement.

- Le troisième essai concerne une perturbation de la forme :

$$
d_{7}(t)=A \cdot\left[\sin \left(\omega_{1} t\right)+\sin \left(\omega_{2} t\right)+\sin \left(\omega_{3} t\right)\right]
$$

où les pulsations correspondent aux 3 premiers modes propres, c'est-à-dire $\omega_{1}=87 \mathrm{rad} / \mathrm{s} ; \omega_{1}=120 \mathrm{rad} / \mathrm{s} ; \omega_{1}=150.5 \mathrm{rad} / \mathrm{s}$. Nous présentons à la figure 17 les résultats expérimentaux obtenus.

REMARQUE. - Le rejet de la perturbation est presque immédiat. L'efficacité est moyenne (réduction $65 \%$ pour la sortie $y_{4}$ et $50 \%$ pour la sortie $y_{5}$ ).
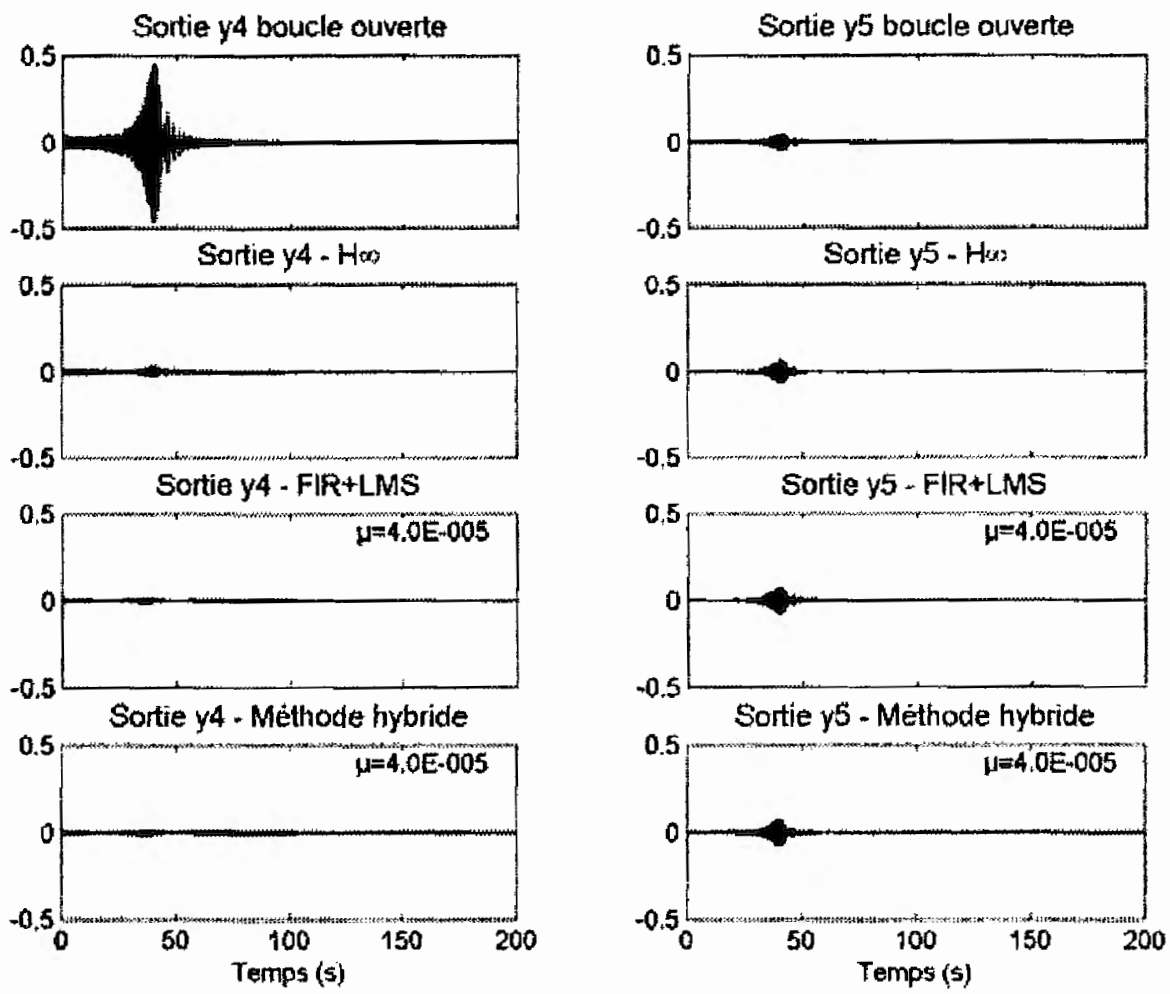

Figure 16. Expérimentations à fréquence variable 

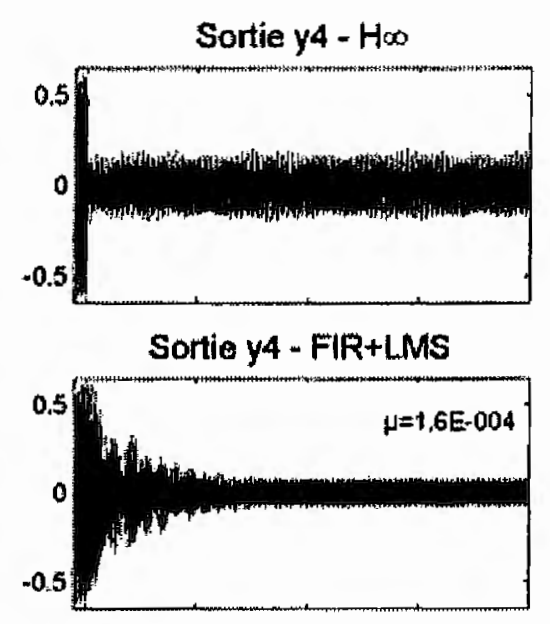

Sortie y4 - Méthode hybride

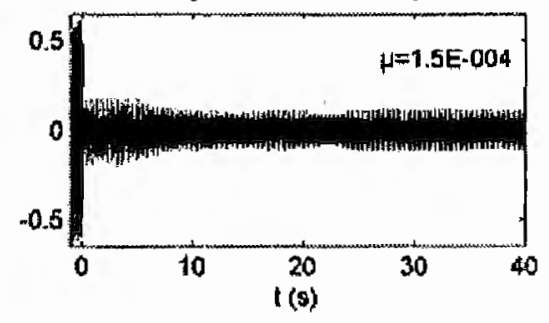

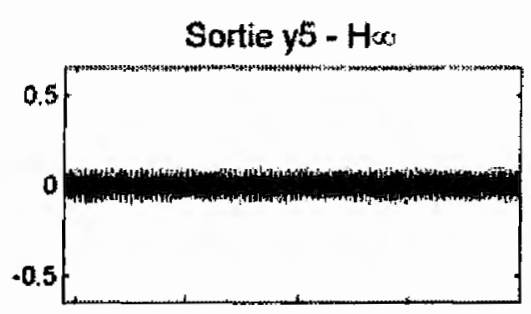

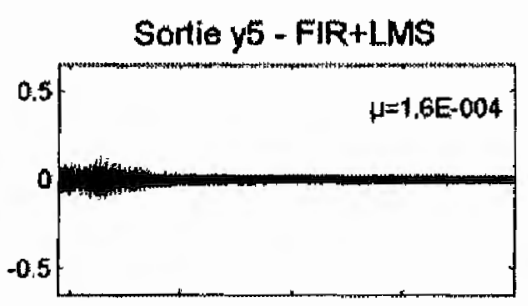

Sortie y5 - Méthode hybride

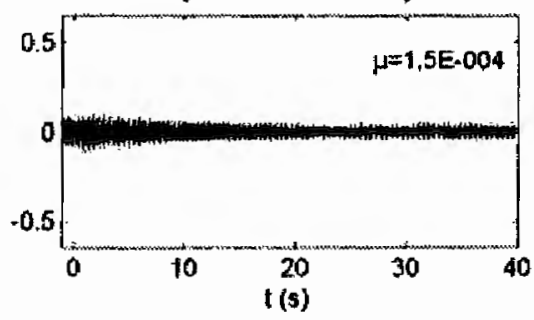

Figure 17. Expérimentations pour une perturbation périodique

\section{Conclusions}

Dans cet article, nous avons montré que :

- Le rejet de perturbations sur des structures souples soulève une grande quantité de problèmes : choix du modèle et de son ordre, choix du type de représentation, etc.

- Le choix de la méthode de rejet des perturbations dépend de leur nature. Dans de nombreux cas industriels, les perturbations sont sinusoïdales (ou composées de plusieurs sinusö̈des). Dans ce cas, la méthode mixte nous semble la meilleure. Cependant, sa mise en œuvre, bien qu'elle ne présente pas de difficulté majeure, doit être effectuée avec soin, car ces types de commande reposent sur une bonne connaissance des fonctions de transfert manipulées. Il est donc nécessaire d'identifier le système avec le plus de précision possible. Notons que certains signaux de perturbation se prêtent mal au traitement adaptatif : c'est le cas par exemple des perturbations non périodiques ou des chocs sur la structure.

- L'intérêt de la commande mixte vient de ce que le régulateur Ho assure une marge de stabilité convenable alors que le filtre adaptatif réagit immédiatement à l'apparition d'une vibration.

- Le rejet peut être effectué avec une commande adaptative multivariable. Des études (non présentées ici) montrent que le rejet s'effectue de manière plus efficace si on augmente le nombre de capteurs et d'actionneurs. Toutefois, la multiplication des actionneurs et des capteurs augmente le coût de l'instrumentation. 


\section{Bibliographie}

[BRU 99] BRUANT I., Coffignal G., Lene, F., Verge M., "Methodology for determination of piezoelectric actuators and sensors geometry on beams structures", Journal of Sound and Vibration, vol. 219, $\mathrm{n}^{\circ}$ 5, 1999.

[ELL 87] Elliot S.J., Stothers I.M., Nelson P.A., "A Multiple Error LMS Algorithm and Its Application to the Active Control of Sound and Vibration", IEEE Transactions on Acoustics, Speech, and Signal Processing, 1987.

[ERI 92] ERIKSSON L.J., Active Noise Control, Didisonix, 1992.

[GAW 98] GAWRONSKI W.K., Dynamics and control of structures, a modal approach, Springer, 1998.

[GER 95] GERADIN M, VIXEN D., Théorie des vibrations, application à la dynamique des structure's, Masson, 1995.

[HEN 99] Henriot P., Vergé M., Coffignal G, "Model reduction for active vibration control", SPIE's $7^{\text {th }}$ Annual International Symposium on Smart Structures and Materials, 1999, Newport Beach, Californie, USA.

[HEN 00] HeNRIOT P., Contribution au Contrôle Actif des Structures, Thèse ENSAM, 2000.

[JAU 91] JAUME D., Thelliez S., Verge M, Commande des systèmes dynamiques par calculateur, Eyrolles, 1991

[JIA 97] Jiang F., Tsuji H., OhmoRi H., Sano A., "Adaptation for Active Noise Control", IEEE Control Systems, 12, 1997.

[KUO 96] Kuo S.M., MoRGan D.R., Active Noise Control Systems, Editions Wiley, 1996.

[LEE 02] LEE Y.Y., YUEN K.K., CHENG C.F., "Numerical simulation model of vibration responses of rectangular plates embeded with piezoelectric actuators", Thin Walled Structures, $\mathrm{n}^{\circ} 40,2002$, p. 1-28.

[LJU 87] LJUNG L., System Identification - Theory for the user, Prentice-Hall Englewood Cliffs, N.J., 1987.

[MAC 95] MACCHI O., Adaptive Processing, Editions Wiley 1995.

[MAU 92] Maugin G.A., Pouget J., Drouot R., Collet B., Non linear electromechanical couplings, Editions John Wiley, 1992.

[QUE 94] QUETIN F., Recalage de Modèles Eléments Finis en Dynamique des Structures, Thèse ENSAM, 1994.

[SKO 96] Skogestad S., Postlewaite I., Multivariable Feedback control, Editions Wiley 1996.

[WID 85] WIdrow B., STEarns S. D., Adaptive Signal Processing, Prentice-Hall, 1985. 